\title{
Semiclassical Quantization Rules Near Separatrices
}

\section{Pavel M. Bleher}

School of Natural Sciences, Institute for Advanced Study, Princeton, NJ 08540, USA

Received: 15 October 1993/in revised form: 14 December 1993

Abstract: We derive semiclassical quantization equations with uniform estimate of the error term near unstable equilibria of the classical system for the one-dimensional Schrödinger operator.

\section{Introduction}

The Einstein-Brillouin-Keller (EBK) quantization rule gives semiclassical energy levels of a quantized completely integrable classical system with $n$ degrees of freedom as

$$
E\left(k_{1}, \ldots, k_{n}\right)=H\left(\pi \hbar\left(k_{1}+\left(\alpha_{1} / 4\right)\right), \ldots, \pi \hbar\left(k_{n}+\left(\alpha_{n} / 4\right)\right)\right),
$$

where $k_{1}, \ldots, k_{n}$ are integer quantum numbers and $\alpha_{1}, \ldots, \alpha_{n}$ are the Maslov indices (see, e.g., [BT]). Here $H\left(I_{1}, \ldots, I_{n}\right)$ is the classical Hamilton function in action-angle coordinates $I_{1}, \ldots, I_{n} ; \varphi_{1}, \ldots, \varphi_{n}$. For a one-dimensional potential hole $U(x)$, (1.1) reduces to the Bohr-Sommerfeld quantization rule of the old quantum mechanics,

$$
\hbar^{-1} \int_{x_{-}}^{x_{+}} \sqrt{2 m\left(E_{k}-U(x)\right)} d x=\pi(k+(1 / 2)) .
$$

The EBK quantization rule has been established in a number of cases (see [CdV, Laz and KMS]). Still many problems remain open and among them the most important problems are, probably, the following two:

(i) Does the EBK quantization rule satisfy the correspondence principle, which means that (1.1) gives all (or at least almost all except finitely many) quantum energy levels?

(ii) What is a uniform quantization rule near separatrices?

In the present paper we address ourselves mostly the second problem in the simplest, one-dimensional case. 
Consider a classical particle which moves on a circle of the length $L$ in the field created by a smooth potential $U(x)$. Assume that $x=0$ is the only absolute maximum of $U(x)$. Then if the energy $E$ of the particle is bigger than $U_{\max }=U(0)$, the particle will run around the circle with some average nonzero velocity. On the other hand, if the energy of the particle is less than $U_{\max }$ the particle cannot cross the point $x=0$ and it will oscillate between two turning points $x_{ \pm}$which satisfy $U\left(x_{ \pm}\right)=E$. For $E<U_{\max }$ the quantized energy levels are determined (up to $O\left(\hbar^{2}\right)$ ) by (1.2), while for $E>U_{\max }$ the quantization rule is

$$
\hbar^{-1} \int_{0}^{L} \sqrt{2 m\left(E_{2 k-1,2 k}-U(x)\right)} d x=2 \pi k .
$$

Thus the quantization rule changes discontinuously when $E=E_{k}$ passes the value $U_{\max }$ and this implies that the error term $O\left(\hbar^{2}\right)$ in the value of energy levels given by the quantization rules (1.2), (1.3) cannot be uniform when $E \rightarrow U_{\max }$. Our aim in the present paper is to derive a quantization rule with uniform estimate of the error term, which connects (1.2) to (1.3).

A similar situation appears when $E$ passes the value of a local maximum of $U(x)$. Consider a double-well potential with a local maximum at $x=0$ (see Fig. 1). Then for $E<U(0)$ we have independent quantization rules for each well,

$$
\hbar^{-1} \int_{x_{-}^{\prime}}^{x_{-}^{\prime}} \sqrt{2 m(E-U(x))} d x=\pi\left(k_{-}+(1 / 2)\right),
$$

and

$$
\hbar^{-1} \int_{x_{+}^{\prime}}^{x_{+}} \sqrt{2 m(E-U(x))} d x=\pi\left(k_{+}+(1 / 2)\right),
$$

while $E>U(0)$ the quantization rule is (1.2). Again the quantization rule changes discontinuously at $U(0)$ and so the error term $O\left(\hbar^{2}\right)$ is not uniform when $E \rightarrow U(0)$. Our aim is to derive a quantization rule with uniform estimate of the error term, which connects (1.2) to (1.4), (1.5). Let us turn to exact formulation of our results.

Consider a one-dimensional Schrödinger equation on $[-(L / 2),(L / 2)]$,

$$
\begin{aligned}
-\frac{\hbar^{2}}{2 m} \frac{d^{2} \psi}{d x^{2}}+U \psi & =E \psi, \\
\psi(x+L) & =\psi(x),
\end{aligned}
$$

with periodic boundary conditions, where $U=U(x)$ is a periodic $C^{\infty}$ potential, $U(x+L)=U(x)$, with a nondegenerate absolute maximum at $x=0$ :

$$
U_{\max }=U(0)>U(x) \quad \text { if } x \neq 0 ; \quad U^{\prime \prime}(0)<0 .
$$

Let $E_{0} \leqq E_{1} \leqq E_{2} \leqq \ldots$ be eigenvalues (quantum energy levels) of (1.6) and

$$
I(E)=\hbar^{-1} \int_{\{x: E \geqq U(x)\}} \sqrt{2 m(E-U(x))} d x .
$$

We are interested in eigenvalues $E=E_{k}$ near $U_{\max }$ and we prove the following result: 


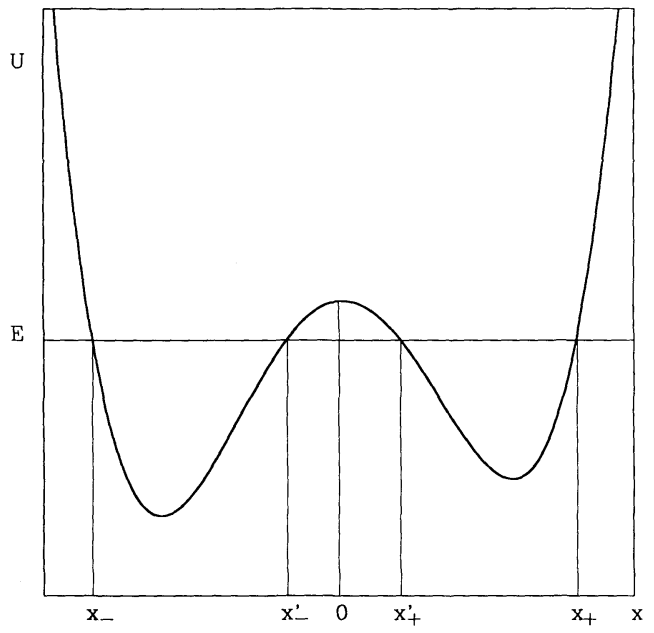

Fig. 1. Double-well potential

Theorem 1.1. There exists $\varepsilon>0$ such that in the interval $U_{\max }-\varepsilon<E<U_{\max }+\varepsilon$, $E=E_{2 k}, E_{2 k-1}$ are, up to $O\left(\hbar^{4 / 3}\right)$, solutions of the equation

$$
I(E)=\pi\left[2 k+\gamma_{ \pm}(a(E))\right]
$$

where $a(E)=\hbar^{-1}\left(m /\left|U^{\prime \prime}(0)\right|\right)^{1 / 2}(E-U(0))$ and

$$
\gamma_{ \pm}(t)=\pi^{-1}\left[\arg \Gamma((1 / 2)+i t)-t \ln |t|+t \pm \arctan e^{-\pi t}\right]
$$

Remark. We in fact prove that $E=E_{2 k}, E_{2 k-1}$ are solutions of Eq. (1.7) up to $O\left(\min \left\{\hbar^{4 / 3}, \tau \hbar^{2}\right\}\right)$ where $\tau=1+\left|U^{\prime \prime \prime}(0)\right||U(0)-E|^{-1 / 2}$, which gives $O\left(\hbar^{2}\right)$ when $U^{\prime \prime \prime}(0)=0$ (e.g., for even $U$ ).

The graphs of the functions $\gamma_{ \pm}(t)$ are shown on Fig. 2. Observe that $\gamma_{ \pm}(\infty)=0$ so for $E-U_{\max } \gg \hbar\left(\mid U^{\prime \prime}(0) / m\right)^{1 / 2},(1.7)$ reduces to

$$
\hbar \int_{-L / 2}^{L / 2} \sqrt{2 m(E-U(x))} d x=2 \pi k,
$$

and $\gamma_{ \pm}(-\infty)= \pm(1 / 2)$, so for $U_{\max }-E \gg \hbar\left(\left|U^{\prime \prime}(0)\right| / m\right)^{1 / 2},(1.7)$ reduces to

$$
\hbar^{-1} \int_{\{x: E \geqq U(x)\}} \sqrt{2 m(E-U(x))} d x=\pi[2 k \pm(1 / 2)],
$$

which is equivalent to (1.2). Equations (1.9) and (1.10) are the Bohr-Sommerfeld quantization rules without and with turning points, respectively, and (1.7) describes a continuous transition from (1.9) to (1.10) with a uniform estimate of the error term.

Consider now the case when $x=0$ is a local and not a global maximum of $U(x)$ ("double-well" potential, see Fig. 1). Define for $E \leqq U(0)$, the points $x_{-}<x_{-}^{\prime} \leqq$ $x_{+}^{\prime}<x_{+}$as solutions of the equation $U(x)=E$. For $E \geqq U(0)$ we define $x_{-}<x_{+}$as solutions of $U(x)=E$ and $x_{-}^{\prime}=x_{+}^{\prime}=0$. For the sake of simplicity we will assume 


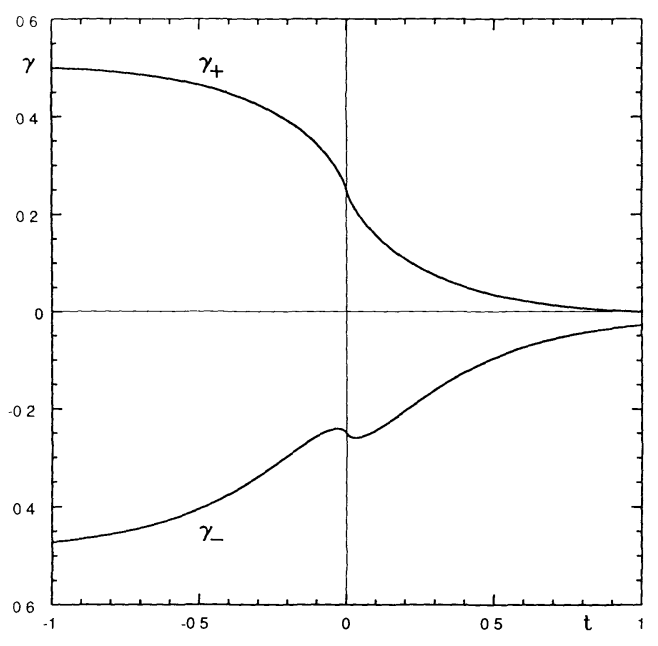

Fig. 2. Graphs of the functions ${ }^{\prime} \pm \pm$

that in some interval $|E-U(0)| \leqq \varepsilon_{0}, \varepsilon_{0}>0$, there are no other solutions of the equation $U(x)=E$. Let

$$
I_{1}(E)=\hbar^{-1} \int_{x_{-}}^{x_{-}^{\prime}} \sqrt{2 m(E-U(x))} d x
$$

and

$$
I_{2}(E)=\hbar^{-1} \int_{x_{+}^{\prime}}^{x_{+}} \sqrt{2 m(E-U(x))} d x .
$$

We will take the convention that $\tan x+\tan y=2 \tan z$ if either $\tan x, \tan y, \tan z$ are finite and the equation holds or at least two of these three numbers are infinite.

Theorem 1.2. There exists $\varepsilon>0$ such that in the interval $U(0)-\varepsilon<E<U(0)+\varepsilon$, all the eigenvalues $E_{k}$ of (1.6) can be found, with the error $O\left(\hbar^{4 / 3}\right)$, as solutions of the equation

$$
\tan \left(I_{1}-\phi_{+}+(\pi / 4)\right)+\tan \left(I_{2}-\phi_{+}+(\pi / 4)\right)=2 \tan \left(\phi_{-}-\phi_{+}\right),
$$

with $I_{1,2}=I_{1,2}(E)$ and $\phi_{ \pm}=(\pi / 2) \gamma_{ \pm}(a(E))$, where $a(E)$ and $\gamma_{ \pm}(t)$ are the same as in Theorem 1.1.

When $E-U(0) \gg \hbar\left(\left|U^{\prime \prime}(0)\right| / m\right)^{1 / 2}, \phi_{-}$and $\phi_{+}$are close to 0 and (1.13) reduces to the Bohr-Sommerfeld equation (1.2). When $U(0)-E \gg \hbar\left(\left|U^{\prime \prime}(0)\right| / m\right)^{1 / 2}, \pm \phi_{ \pm}$is close to $\pi / 4$, so that $\left|\tan \left(\phi-\phi_{+}\right)\right|$is close to infinity and (1.13) reduces to two possibilities described by (1.4), (1.5). In the case of a symmetric double-well potential $U(x), I_{1}=I_{2}$ and (1.13) reduces to the equation

$$
I_{1}=I_{2}=\pi k+\phi_{ \pm} \pm(\pi / 4) \text {. }
$$

In general, other wells can exist in which the particle have energy close to $U(0)$. In this case (1.13) represents the energy levels with eigenstates (or quasi-eigenstates) localized in the double well area under consideration, and there are other energy levels near $U(0)$ with (quasi-)eigenstates localized in the other wells. 
It is to be noted that near $U(0)$ the energy levels are more dense than on the average. Consider, for instance, the case when $x=0$ is the absolute maximum of the potential $U(x)$ such that $U(x)<U(0)$ for $x \neq 0$ and $U^{\prime \prime}(0) \neq 0$. Define

$$
b(E)=\int_{\{x \cdot E \geqq U(x)\}} \sqrt{E-U(x)} d x .
$$

Then it is not difficult to show that as $t \rightarrow 0$,

$$
b(U(0)+t)=b_{0}(t)+t|\log | t|| b_{1}(t),
$$

where $b_{0}(t)$ and $b_{1}(t)$ are $C^{\infty}$ functions and $b_{1}(0)=\left|2 U^{\prime \prime}(0)\right|^{-1 / 2}$. Comparing this asymptotics with the quantization equation (1.7), we obtain that for energy levels $E_{k}$ lying in $O(\hbar)$-neighborhood of $U(0)$ the spacing between neighboring energy levels is of order of const $\hbar|\log \hbar|^{-1}$, while on the average it is of order of const $\hbar$. Similar asymptotics of the spacing between neighboring energy levels holds also in $O(\hbar)$-neighborhood of any local maximum of $U(x)$.

The asymptotic expansions for the one-dimensional Schrödinger equation is a highly advanced area of investigations (see excellent reviews in [BM] and [Olv3] with a lot of references therein). So it was very surprising to the author that the semiclassical quantization rules with uniform estimate of the error term, derived in the present work were seemingly not known before. A very close to (1.7) quantization condition was recently obtained in the paper [KMS] of Kosygin, Minasov and Sinai for the Laplace-Beltrami operators on the Liouville surfaces. Also the asymptotic formulas describing wave penetration through a potential barrier (see $[\mathrm{BM}, \mathrm{Olv} 3]$ and references therein) are related to the present study. The importance of the phases $\gamma_{ \pm}$for these problems was emphasised in the works [FHWW, Mil] and others. For analytic double-well potentials N. Fröman, P.O. Fröman, Muhrman and Paulsson (see [FFMP] and references therein) derived, without estimation of the error term, a semiclassical quantization formula which includes integration over different paths in complex plane (the author thanks Michael Berry for calling his attention to this work). It can be shown that this formula is consistent with (1.13) in the limit when $E \rightarrow U(0)$.

We will prove Theorem 1.1 and 1.2 in the next section. The proof uses the matching of asymptotic solutions of the Schrödinger equation (1.6) constructed near different turning points.

\section{Proof of Theorems}

Proof of Theorem 1.1. To construct approximate solutions of the Schrödinger equation (1.6) we use the method of comparison equations which was first set out by Miller and Good [MG] and Dingle [Din] (see also papers [HM] of Hecht and Mayer and [Mor] of Moriguchi). A very good exposition of the method of comparison equations and other relevant methods and applications is given in the review article $[\mathrm{BM}]$ of Berry and Mount.

A model (or comparison) equation for (1.6), when $E$ is near $U_{\max }$, is the Weber equation

$$
v^{\prime \prime}+\left(a+\left(x^{2} / 4\right)\right) v=0 .
$$


Let $v_{ \pm}(x ; a)$ be even and odd real valued solutions of the Weber equation. We look for the WKB solutions of (1.6) in the form

$$
\psi_{ \pm}(x)=\frac{1}{\sqrt{\varphi^{\prime}(x)}} v_{ \pm}\left(\hbar^{-1 / 2} \varphi(x) ; \hbar^{-1} a\right),
$$

where $\varphi(x)$ is an unknown function. Substituting the form (2.2) into (1.6) we obtain the equation

$$
\left(\varphi^{\prime}\right)^{2}\left(a+\left(\varphi^{2} / 4\right)\right)=2 m(E-U)+\hbar^{2} S\left(\varphi^{\prime}\right)
$$

where

$$
S(\psi)=\left(\psi^{\prime \prime} / \psi\right)-(3 / 2)\left(\psi^{\prime} / \psi\right)^{2}
$$

is the Schwarzian. Let us drop the last term in (2.3) and consider the equation

$$
\left(\varphi^{\prime}\right)^{2}\left(a+\left(\varphi^{2} / 4\right)\right)=2 m(E-U) .
$$

Here $\varphi(x)$ and $a$ are unknown. We claim that we can find such an $a$ that (2.5) has a nice $C^{\infty}$ solution $\varphi(x)$ with $\varphi^{\prime}>0$. Assume first that $E<U_{\max }$. Let $U\left(x_{-}\right)=U\left(x_{+}\right)=E$, so that $E-U(x)<0$ when $x_{-}<x<x_{+}$. Then from (2.5)

$$
a+\left(\varphi^{2}\left(x_{ \pm}\right) / 4\right)=0 \text {, }
$$

so $a<0$. Let us rewrite $(2.5)$ on $x_{-}<x<x_{+}$as

$$
\varphi^{\prime} \sqrt{-a-\left(\varphi^{2} / 4\right)}=\sqrt{2 m(U-E)} .
$$

Integrating from $x_{-}$to $x_{+}$we obtain

$$
\int_{-2|a|^{1 / 2}}^{2|a|^{1 / 2}} \sqrt{-a-\left(\varphi^{2} / 4\right)} d \varphi=\int_{\varphi\left(x_{-}\right)}^{\varphi\left(x_{+}\right)} \sqrt{-a-\left(\varphi^{2} / 4\right)} d \varphi=\int_{x_{-}}^{x_{+}} \sqrt{2 m(U-E)} d x .
$$

The LHS integral is equal to $\pi(-a)$, hence

$$
a=-\pi^{-1} \int_{x_{-}}^{x_{+}} \sqrt{2 m(U-E)} d x .
$$

Lemma 2.1. $a=a(E)$ is $a C^{\infty}$ function of $E$ on $\left[U_{\max }-\varepsilon, U_{\max }\right]$, and when $E \rightarrow U_{\max }$,

$$
a(E)=m^{1 / 2}\left|U^{\prime \prime}(0)\right|^{-1 / 2}\left(E-U_{\max }\right)+O\left(\left|E-U_{\max }\right|^{2}\right) .
$$

Proof of this and all subsequent lemmas is given in the next section.

Observe that (2.8) determines $a=a(E)$ for $E \leqq U_{\max }$. Consider an arbitrary $C^{\infty}$ continuation of $a=a(E)$ to $U_{\max } \leqq E \leqq U_{\max }+\varepsilon$ with $a^{\prime}(E)>0$. Consider the equation

$$
\varphi^{\prime}\left|a+\left(\varphi^{2} / 4\right)\right|^{1 / 2}=|2 m(E-U)|^{1 / 2},
$$

which is equivalent to (2.5) if we assume that $\varphi^{\prime}>0$. Define

$$
I(y ; a)=\int_{y+}^{y}\left|a+\left(\xi^{2} / 4\right)\right|^{1 / 2} d \xi
$$


with $y_{+}=0$ if $a \geqq 0$ and $y_{+}=2|a|^{1 / 2}$ if $a<0$. Define also

$$
J(x ; E)=\int_{x_{+}}^{x}|2 m(E-U(\xi))|^{1 / 2} d \xi,
$$

where $x_{+}=0$ if $E \geqq U_{\max }$ and $x_{+}>0$ is the root of the equation

$$
E-U(x)=0,
$$

if $E<U_{\max }$. Then (2.10) can be rewritten as

$$
(I(\varphi ; a))^{\prime}=(J(x ; E))^{\prime},
$$

hence $I(\varphi ; a)=J(x ; E)+$ const. To have a smooth $\varphi$ we put const $=0$, so that we are looking for $\varphi$ as a solution of the equation

$$
I(\varphi ; a)=J(x ; E) .
$$

Since $I(y ; a)$ is a strictly increasing function $y$ with

$$
\lim _{y \rightarrow \pm \infty} I(y ; a)= \pm \infty
$$

(2.13) has a unique solution $\varphi=\varphi(x ; E)$ which satisfies $(2.10)$ as well. We will consider the solution $\zeta=\zeta(x ; E)$ on the covering line $-\infty<x<\infty$ and not on the circle because $\zeta$ does not satisfy in general the periodic boundary conditions.

Lemma 2.2. Assume that $a=a(E)$ is chosen as described above. Then $\varphi(x ; E)$ is $C^{\infty}$ smooth in $x \in[-(2 / 3) L,(2 / 3) L]$ and for all $x \in[-(2 / 3) L,(2 / 3) L]$ and $E \in$ $\left[U_{\max }-\varepsilon, U_{\max }+\varepsilon\right]$,

$$
\varphi^{\prime}(x ; E)>\lambda>0, \quad \varphi^{\prime}=\partial \varphi / \partial x
$$

and

$$
\left|\varphi^{\prime \prime}(x ; E)\right| \leqq C, \quad\left|\varphi^{\prime \prime \prime}(x ; E)\right| \leqq C \tau, \quad \tau=\left(1+\left|U^{\prime \prime \prime}(0)\right|\left|U_{\max }-E\right|^{-1 / 2}\right) .
$$

It is to be noted that (2.7) implies that when $a<0$,

$$
I\left(2|a|^{1 / 2}\right)-I\left(-2|a|^{1 / 2}\right)=J\left(x_{+} ; E\right)-J\left(x_{-} ; E\right),
$$

hence Eq. (2.13) is equivalent to

$$
I_{-}(\varphi ; a)=J_{-}(x ; E),
$$

where

$$
I_{-}(\varphi ; a)=\int_{-2|a|^{1 / 2}}^{\varphi} \mid a+\left(\xi^{2} / 4\right)^{1 / 2} d \xi
$$

and

$$
J_{-}(x ; E)=\int_{x_{-}}^{x}|2 m(E-U(\xi))|^{1 / 2} d \xi .
$$

Observe that the even and odd solutions $v_{ \pm}(y ; a)$ of the Weber equation are determined up to constant factors. These factors can be chosen in such a way that the following asymptotics hold: 
Lemma 2.3. When $y \geqq 2\left(a^{2}+0.01\right)^{1 / 4}$,

$$
\begin{aligned}
& v_{+}(y ; a)=\frac{1}{\sqrt{\Phi^{\prime}(y ; a)}} \cos \left(\Phi(y ; a)-\phi_{+}(a)\right), \\
& v_{-}(y ; a)=\frac{1}{\sqrt{\Phi^{\prime}(y ; a)}} \sin \left(\Phi(y ; a)-\phi_{-}(a)\right), \quad \Phi^{\prime}=\partial \Phi / \partial y,
\end{aligned}
$$

where

$$
\phi_{ \pm}(a)=(1 / 2)\left[\arg \Gamma((1 / 2)+i a)-a \ln |a|+a \pm \arctan e^{-\pi a}\right],
$$

and $\Phi(y ; a)$ is a real valued $C^{\infty}$ function such that $\Phi^{\prime}(y ; a)>0$ and

$$
\Phi(y ; a)=I((y ; a)+r(y ; a),
$$

with

$$
\left|d^{j} r(y ; a) / d y^{j}\right| \leqq C y^{-2-j}, \quad j=0,1,2 .
$$

In addition, the $k$-th zero $y=y_{k, \pm}>0$ of $v_{ \pm}(y ; a)$ is the solution of the equation

$$
\begin{aligned}
& \Phi(y ; a)-\phi_{+}(a)=(k-(1 / 2)) \pi, \quad y=y_{k,+}, \\
& \Phi(y ; a)-\phi_{-}(a)=k \pi, \quad y=y_{k,-},
\end{aligned}
$$

respectively.

Lemma 2.3 is well known so we will not prove it (the asymptotics of the function $v_{ \pm}(y ; a)$ as $a \rightarrow \infty$ was studied by Schwid [Sch] who used general results of Langer [Lan] and by Olver, see [Olv1-Olv3] and references therein).

Lemma 2.3 enables us to find the asymptotics of $\psi_{ \pm}(x)=\psi_{ \pm}(x ; E)$ in $(2.2)$ as $\hbar \rightarrow 0$. For the sake of definiteness we will consider $\psi_{+}(x ; E)$. Assume that for all $E \in\left[U_{\max }-\varepsilon, U_{\max }\right]$ the roots $x_{ \pm}$of the equation $E-U(x)=0$ lie in the interval $[-L / 8, L / 8]$. Let $x>L / 4$. Then

$$
\begin{aligned}
\psi_{+}(x ; E) & =\frac{1}{\sqrt{\Phi^{\prime}\left(\hbar^{-1 / 2} \varphi(x ; E) ; \hbar^{-1} a\right) \varphi^{\prime}(x ; E)}} \cos \left(\Phi\left(\hbar^{-1 / 2} \varphi(x ; E) ; \hbar^{-1} a\right)-\phi_{+}(a)\right) \\
& =\frac{\hbar^{1 / 4}}{\sqrt{\Lambda^{\prime}(x ; E)}} \cos \left(\hbar^{-1} \Lambda(x ; E)-\phi_{+}(a)\right),
\end{aligned}
$$

where

$$
\Lambda(x ; E)=\hbar \Phi\left(\hbar^{-1 / 2} \varphi(x ; E) ; \hbar^{-1} a\right), \quad a=a(E) .
$$

By (2.18)

$$
\begin{aligned}
\Lambda(x ; E) & =\hbar I\left(\hbar^{-1 / 2} \varphi(x ; E) ; \hbar^{-1} a\right)+\hbar r\left(\hbar^{-1 / 2} \varphi(x ; E) ; \hbar^{-1} a\right), \\
& =I(\varphi(x ; E) ; a)+\hbar r\left(\hbar^{-1 / 2} \varphi(x ; E) ; \hbar^{-1} a\right),
\end{aligned}
$$

hence by (2.13)

$$
\Lambda(x ; E)=\int_{x_{+}}^{x} \mid 2 m\left(E-\left.U(\xi)\right|^{1 / 2} d \xi+\hbar r\left(\hbar^{-1 / 2} \varphi(x ; E) ; \hbar^{-1} a\right)\right.
$$

Due to (2.19)

$$
\left|d^{j}\left(\hbar r\left(\hbar^{-1 / 2} \varphi(x ; E) ; \hbar^{-1} a\right)\right) / d x^{j}\right| \leqq C \hbar^{2}, \quad j=0,1,2,
$$


hence $(2.20)$ leads to

$$
\psi_{+}(x ; E)=\frac{\hbar^{1 / 4}}{\sqrt{p(x ; E)+O\left(\hbar^{2}\right)}} \cos \left(\hbar^{-1} \int_{x_{+}}^{x} p(\xi ; E) d \xi-\phi_{+}(a)+O(\hbar)\right.
$$

where

$$
p(x ; E)=\sqrt{|2 m(E-U(x))|} .
$$

For $x<-L / 4$ we have a similar formula,

$$
\psi_{+}(x ; E)=\frac{\hbar^{1 / 4}}{\sqrt{p(x ; E)+O\left(\hbar^{2}\right)}} \cos \left(\hbar^{-1} \int_{x_{-}}^{x} p(\xi ; E) d \xi+\phi_{+}(a)+O(\hbar) .\right.
$$

Now we want to glue $\psi_{+}(x ; E)$ at $x=-L / 2$ and at $x=L / 2$ to have a periodic function. Comparing (2.23) with (2.24) we see that modulo error terms of order $O\left(\hbar^{2}\right)$ the periodicity of $\psi_{+}(x ; E)$ holds when

$$
\hbar^{-1} \int_{x_{+}}^{x} p(\xi ; E) d \xi-\phi_{+}(a)=\hbar^{-1} \int_{x_{-}}^{x-L} p(\xi ; E) d \xi+\phi_{+}(a)+2 \pi k, \quad k \in \mathbf{Z}
$$

or, equivalently,

$$
(\pi \hbar)^{-1} \int_{x_{+}}^{x_{-}+L} p(\xi ; E) d \xi=2 k+\gamma_{+}(a), \quad k \in \mathbf{Z}
$$

with

$$
\gamma_{+}(a)=(2 / \pi) \phi_{+}(a)=(1 / \pi)\left[\arg \Gamma((1 / 2)+i a)-a \ln |a|+a+\arctan e^{-\pi a}\right] .
$$

Assuming (2.26) we construct now a periodic approximation $\psi_{+}^{(\text {per) }}(x ; E)$ of $\psi_{+}(x ; E)$.

Let $\chi(x)$ be a $C^{\infty}$ function such that $\chi(x)=0$ when $x \leqq-L / 8, \chi(x)=1$ when $x \geqq L / 8$ and $0 \leqq \chi(x) \leqq 1$ everywhere. Define the function

$$
\psi_{+}^{(\text {per })}(x ; E)=(1-\chi(x-(L / 2))) \psi_{+}(x ; E)+\chi(x-(L / 2)) \psi_{+}(x-L ; E)
$$

on $[0, L]$, which is a $C^{\infty}$ periodic function, since it coincides with $\psi_{+}(x ; E)$ in the interval $0 \leqq x \leqq(3 / 8) L$ and with $\psi_{+}(x-L)$ in the interval $(5 / 8) L \leqq x \leqq L$.

For what follows the following simple lemma will be useful:

Lemma 2.4. $\psi=\psi_{+}(x ; E)$ satisfies the equation

$$
-\frac{\hbar^{2}}{2 m} \frac{d^{2} \psi}{d x^{2}}+\left(U+\frac{\hbar^{2}}{2 m} S\left(\varphi^{\prime}\right)\right) \psi=E \psi
$$

Since $\psi_{+}^{(\text {per })}(x ; E)$ coincides with $\psi_{+}(x ; E)$ in the interval $|x| \leqq(3 / 8) L$, we have: Corollary. $\psi_{+}^{(\text {per })}(x ; E)$ satisfies $E q .(2.29)$ in the interval $|x| \leqq(3 / 8) L$.

When $|x| \geqq(3 / 8) L, \psi_{+}^{(\text {per })}(x ; E)$ satisfies a more complicated equation due to the terms which appear under differentiation of the function $\chi$ in (2.28). In this case we have 
Lemma 2.5. On the interval $(3 / 8) L<x<(5 / 8) L, \psi=\psi_{+}^{(\text {per })}(x ; E)$ satisfies the equation

$$
-\frac{\hbar^{2}}{2 m} \frac{d^{2} \psi}{d x^{2}}+\left(U+\frac{\hbar^{2}}{2 m} S\left(\varphi^{\prime}\right)\right) \psi=E \psi+r .
$$

where

$$
|r(x)| \leqq C \hbar^{3}\|\psi\|, \quad\|\cdot\|=\|\cdot\|_{L^{2}} .
$$

Let $H$ be the operator

$$
\psi \rightarrow H \psi=-\frac{\hbar^{2}}{2 m} \frac{d^{2} \psi}{d x^{2}}+U \psi
$$

in $L^{2}([-L / 2, L / 2])$ with periodic boundary conditions.

Lemma 2.6. Assume that $E$ satisfies (2.26). Then $\psi=\psi_{+}^{(\mathrm{per})}(x ; E)(x ; E)$ satisfies

$$
\|(H-E) \psi\| \leqq C \tau \hbar^{2}\|\psi\|, \quad \tau=\left(1+\left|U^{\prime \prime \prime}(0) \| U_{\max }-E\right|^{-1 / 2}\right),
$$

and

$$
\|\psi\| \geqq C^{-1} \hbar^{1 / 4}, \quad C>0 .
$$

Lemma 2.7. Assume that $E$ satisfies (2.26). Then $\exists E_{m,+}, \psi_{m,+}$ such that

$$
H \psi_{m,+}=E_{m,+} \psi_{m,+}, \quad\left\|\psi_{m,+}\right\|=1,
$$

and

$$
\left|E-E_{m,+}\right| \leqq C \min \left\{\hbar^{4 / 3}, \tau \hbar^{2}\right\} .
$$

In addition, $\psi_{m,+}$ has exactly $2 k$ zeroes and all these zeroes are simple.

A similar statement holds for the - -case, so that if $E$ is a solution of Eq. (2.26) with $\gamma_{-}(a)$ instead of $\gamma_{+}(a)$ then there exist $E_{m,-}, \psi_{m,-}$ such that $H \psi_{m,-}=E_{m,-} \psi_{m,-},\left\|\psi_{m,-}\right\|=1$, and (2.34) holds with the replacement + for - . Also $\psi_{m .}$ - has exactly $2 k$ zeroes and all of them are simple.

Lemma 2.8. $\psi_{m,-}$ can be chosen in such way that $\psi_{m,-}$ and $\psi_{m,+}$ are not collinear.

Observe that if $E_{0} \leqq E_{1} \leqq E_{2} \leqq \ldots$ are eigenvalues of $H$ with eigenfunctions $\psi_{0}, \psi_{1}, \psi_{2}, \ldots$, respectively, then $\psi_{2 k-1}$ and $\psi_{2 k}$ has exactly $2 k$ zeroes and all these zeroes are simple (see, e.g., [Ble]). This proves that $E_{m, \pm}$ coincide with $E_{2 k}, E_{2 k-1}$ and hence $E_{2 k}, E_{2 k-1}$ satisfy (1.7) up to $O\left(\min \left\{\hbar^{4 / 3}, \tau \hbar^{2}\right\}\right)$. Theorem 1.1 is proved.

Proof of Theorem 1.2. We are looking for solutions of (1.6) in the general form

$$
\psi=a \psi_{+}+b \psi_{-},
$$

where $\psi_{ \pm}$are the WKB solutions (2.2). By (2.23) up to a constant factor and an error term

$$
\psi_{+}=[p(x ; E)]^{-1 / 2} \mathfrak{R} \exp \left[i\left(I-\phi_{+}\right)\right], \quad x \geqq L / 4,
$$

where

$$
I=\hbar^{-1} \int_{x_{\perp}^{\prime}}^{x} p(\xi ; E) d \xi
$$


Similarly,

$$
\psi_{-}=[p(x ; E)]^{-1 / 2} \mathfrak{R} \exp \left[i\left(I-\phi_{-}-(\pi / 2)\right)\right], \quad x \geqq L / 4
$$

so

$$
\psi=[p(x ; E)]^{-1 / 2} \mathfrak{R}\left\{a \exp \left[i\left(I-\phi_{+}\right)\right]+b \exp \left[i\left(I-\phi_{-}-(\pi / 2)\right)\right]\right\}, \quad x \geqq L / 4 .
$$

Simple calculations give now

$$
\psi=A[p(x ; E)]^{-1 / 2} \cos (I-\phi)
$$

with

$$
\phi=\phi_{+}+\operatorname{arccot}[(a+b \cos \Delta \phi) /(b \sin \Delta \phi)]
$$

and $\Delta \phi=\phi_{-}+(\pi / 2)-\phi_{+}$. Now we are to glue this formula with the asymptotics which comes from $x_{+}$, which is

$$
\psi=A[p(x ; E)]^{-1 / 2} \cos \left[-\hbar^{-1} \int_{x}^{x_{+}} p(\xi ; E) d \xi+(\pi / 4)\right] .
$$

This leads to the equation $I_{1}=\phi+(\pi / 4)+k \pi$, or

$$
I_{1}-\phi_{+}-(\pi / 4)-k \pi=\operatorname{arccot}[(a+b \cos \Delta \phi) /(b \sin \Delta \phi)] .
$$

By the gluing condition at $x \leqq-L / 4$ we have a similar equation,

$$
I_{2}-\phi_{+}-(\pi / 4)-k^{\prime} \pi=\operatorname{arccot}[(a-b \cos \Delta \phi) /(-b \sin \Delta \phi)] \text {. }
$$

Hence

$$
\left.\cot \left[I_{1,2}-\phi_{+}-(\pi / 4)\right]=(a \pm b \cos \Delta \phi) /( \pm b \sin \Delta \phi)\right]
$$

and

$$
\cot \left[I_{1}-\phi_{+}-(\pi / 4)\right]+\cot \left[I_{2}-\phi_{+}-(\pi / 4)\right]=2 \cot \Delta \phi .
$$

Since $\cot (x-(\pi / 2))=-\tan x$ this is equivalent to

$$
\tan \left[I_{1}-\phi_{+}+(\pi / 4)\right]+\tan \left[I_{2}-\phi_{+}+(\pi / 4)\right]=2 \tan \left(\phi_{-}-\phi_{+}\right) .
$$

This gives (1.13). The remaining part of the proof of Theorem 1.2 is similar to the proof of Theorem 1.1. Theorem 1.2 is proved.

\section{Proof of Lemmas}

Proof of Lemma 2.1. Let $f(x)$ be a smooth function such that

$$
U_{\max }-U(x)=(f(x))^{2}, \quad f(0)=0, \quad f^{\prime}(0)=\sqrt{-U^{\prime \prime}(0) / 2} .
$$

Then

$$
y=\left(U_{\max }-E\right)^{-1 / 2} f(x)
$$

satisfies

$$
U(x)-E=\left(U_{\max }-E\right)\left(1-y^{2}\right)
$$

From (3.1)

$$
x=g\left(\left(U_{\max }-E\right)^{1 / 2} y\right),
$$


where $g(f(x))=x, g^{\prime}(0)=\left(-U^{\prime \prime}(0) / 2\right)^{-1 / 2}$, so

$$
\int_{x_{-}}^{x_{+}} \sqrt{U-E} d x=\left(U_{\max }-E\right) \int_{-1}^{1} \sqrt{1-y^{2}} g^{\prime}\left(\left(U_{\max }-E\right)^{1 / 2} y\right) d y .
$$

Let $g_{ \pm}(t)=(1 / 2)[g(t) \pm g(-t)]$. Then $g_{+}^{\prime}(t)$ is an odd function, so

$$
\int_{-1}^{1} \sqrt{1-y^{2}} g_{+}^{\prime}\left(\left(U_{\max }-E\right)^{1 / 2} y\right) d y=0
$$

while $g_{-}^{\prime}(t)$ is an even function, so $g_{-}^{\prime}(t)=h\left(t^{2}\right)$ with a $C^{\infty}$-smooth $h(t)$ such that

$$
h(0)=g^{\prime}(0)=\left(-U^{\prime \prime}(0) / 2\right)^{-1 / 2} \text {, }
$$

and

$$
\begin{aligned}
\int_{x_{-}}^{x_{+}} \sqrt{U-E} d x & =\left(U_{\max }-E\right) \int_{-1}^{1} \sqrt{1-y^{2}}\left[g_{+}^{\prime}\left(\left(U_{\max }-E\right)^{1 / 2} y\right)+g_{-}^{\prime}\left(\left(U_{\max }-E\right)^{1 / 2} y\right)\right] d y \\
& \left.=\left(U_{\max }-E\right) \int_{-1}^{1} \sqrt{1-y^{2}} h\left(\left(U_{\max }-E\right) y^{2}\right)\right) d y .
\end{aligned}
$$

In virtue of (2.8), this implies that $a(E)$ is a $C^{\infty}$-function of $E$ and when $E \rightarrow U_{\max }$, (2.9) holds. Lemma 2.1 is proved.

Proof of Lemma 2.2 Let first $E<U_{\max }$. We are looking for a smooth solution $\varphi$ of (2.5) with $\varphi^{\prime}>0$, so (2.5) is equivalent to

$$
\begin{gathered}
\varphi^{\prime}\left|a+\left(\varphi^{2} / 4\right)\right|^{1 / 2}=|2 m(E-U)|^{1 / 2}, \\
\varphi\left(x_{ \pm}\right)= \pm 2|a|^{1 / 2} .
\end{gathered}
$$

Let $U_{\max }-U(x)=f(x)^{2}$ where $f(x)$ is a $C^{\infty}$ function with $f^{\prime}(x)>0$, and

$$
\begin{aligned}
y & =\left|U_{\max }-E\right|^{-1 / 2} f(x), \\
\zeta(y ; E) & =(1 / 2)|a|^{-1 / 2} \varphi(x ; E) .
\end{aligned}
$$

Then (3.2) reads

$$
\begin{aligned}
\zeta^{\prime}\left|\zeta^{2}-1\right|^{1 / 2} & =h(y ; E)\left|y^{2}-1\right|^{1 / 2} \\
\zeta( \pm 1) & = \pm 1
\end{aligned}
$$

where

$$
h(y ; E)=(m / 2)^{1 / 2}\left|U_{\max }-E\right||a|^{-1} g^{\prime}\left(\left|U_{\max }-E\right|^{1 / 2} y\right), \quad g(f(x))=x .
$$

By (2.9),

$$
h\left(y ; U_{\max }\right)=(m / 2)^{1 / 2} m^{-1 / 2}\left|U^{\prime \prime}(0)\right|^{1 / 2} g^{\prime}(0)=1,
$$

so that when $E=U_{\max }$ Eq. (3.4) has the evident solution $\zeta=y$. Let $b=\left|U_{\max }-E\right|^{1 / 2}$, so that $E=U_{\max }-b^{2}$, and

$$
G(y ; b)=\int_{1}^{y} h\left(\xi ; U_{\max }-b^{2}\right)\left|\xi^{2}-1\right|^{1 / 2} d \xi .
$$


Then (3.4) implies

$$
G(\zeta)=G(y ; b)
$$

where

$$
G(\zeta)=G(\zeta ; 0)=\int_{1}^{\zeta}\left|\xi^{2}-1\right|^{1 / 2} d \xi
$$

Since $G(\zeta)$ is an increasing function (3.8) determines $\zeta=\zeta(y ; b)$ uniquely. Let us verify that $\zeta$ is smooth in $y$. Consider first $y=1$.

Observe that $G(\zeta)$ has a semicubic singularity at $\zeta=1$ and $G(\zeta)^{2 / 3}$, where we define $x^{2 / 3}$ as $|x|^{2 / 3} \operatorname{sgn} x$, is a $C^{\infty}$ function near $\zeta=1$ with $\left(G(\zeta)^{2 / 3}\right)^{\prime}>0$. Similarly, $G(y ; b)$ has a semicubic singularity at $y=1$, and $G(y ; b)^{2 / 3}$ is a $C^{\infty}$ function near $y=1$ with $\left(G(y ; b)^{2 / 3}\right)^{\prime}>0$. Since Eq. (3.8) can be rewritten as

$$
G(\zeta)^{2 / 3}=G(y ; b)^{2 / 3}
$$

this implies that $\zeta(y ; b)$ is $C^{\infty}$ smooth in $y$ near $y=1$ and $\zeta^{\prime}(y ; b)>0$. Consider second $y=-1$.

The choice of $a$ in (2.8) is equivalent to

$$
(-\pi / 2=) G(-1)=G(-1 ; b) .
$$

(integrate the both sides of (3.4) from -1 to 1 ), and hence (3.8) is equivalent to

$$
(G(\zeta)-G(-1))^{2 / 3}=(G(y ; b)-G(-1 ; b))^{2 / 3} .
$$

The function

$$
(G(y ; b)-G(-1 ; b))^{2 / 3}=\left(\int_{-1}^{y} h\left(\xi ; U_{\max }-b^{2}\right)\left|\xi^{2}-1\right|^{1 / 2} d \xi\right)^{2 / 3}
$$

is $C^{\infty}$ smooth near $y=1$ with $\left((G(y ; b)-G(-1 ; b))^{2 / 3}\right)^{\prime}>0$. Hence from (3.11) we obtain that $\zeta$ is smooth near $y=-1$ with $\zeta^{\prime}>0$. Since, in addition, the function $G(y ; b)$ is smooth and $G^{\prime}(y ; b)>0$ when $y \neq \pm 1,(3.8)$ implies that $\zeta(y ; b)$ is smooth and $\zeta^{\prime}(y ; b)>0$ everywhere outside of $y= \pm 1$, so that $\zeta(y ; b)$ is smooth and $\zeta^{\prime}(y ; b)>0$ everywhere. Let us prove that

$$
\left|\zeta^{\prime \prime}(y ; b)\right|,\left|\zeta^{\prime \prime \prime}(y ; b)\right|<C\left(\left|U^{\prime \prime \prime}(0)\right| b+b^{2}\right), \quad \forall|y| \leqq y_{\max }=\max _{x \in[-L / 2, L / 2]}|y| .
$$

Assume first that $|y| \leqq A$ where $A>1$ is an arbitrary fixed number.

We can rewrite (3.5) as

$$
h(y ; E)=\lambda\left(b^{2}\right) g^{\prime}(b y), \quad b=\left|U_{\max }-E\right|^{1 / 2},
$$

where by Lemma $2, \lambda(t)$ is $C^{\infty}$ positive function on $[0, \varepsilon]$. Then (3.7) reads

$$
G(y ; b)=\lambda\left(b^{2}\right) \int_{1}^{y} g^{\prime}(b \xi)\left|\xi^{2}-1\right|^{1 / 2} d \xi .
$$

Assume $y \geqq 0$ and put $y=1+z, \xi=1+\eta^{2} z$. Then

$$
G(y ; b)=2 \lambda\left(b^{2}\right) z^{3 / 2} \int_{0}^{1} g^{\prime}\left(b\left(1+\eta^{2} z\right)\right)\left(2+\eta^{2} z\right)^{1 / 2} \eta^{2} d \eta,
$$


so that

$$
(G(y ; b))^{2 / 3}=H(z ; b)
$$

with

$$
H(z ; b)=z\left(2 \lambda\left(b^{2}\right) \int_{0}^{1} g^{\prime}\left(b\left(1+\eta^{2} z\right)\right)\left(2+\eta^{2} z\right)^{1 / 2} \eta^{2} d \eta\right)^{2 / 3} .
$$

Putting $b=0$ we obtain

$$
(G(y))^{2 / 3}=H(z) \equiv H(z ; 0) .
$$

Observe that by (3.8),

$$
\zeta(y)=1+H^{-1}(H(z ; b)), \quad z=y-1 .
$$

From (3.17) we have that $H(z ; b)$ is $C^{\infty}$ in $z \geqq 0$ and $b \in\left[0, \varepsilon^{1 / 2}\right]$.

By (3.5),

$$
(f(x))^{2}=U_{\max }-U(x)=-(1 / 2) U^{\prime \prime}(0) x^{2}-(1 / 6) U^{\prime \prime \prime}(0) x^{3}-\ldots,
$$

so that

$$
\begin{aligned}
f(x) & =\left(\left|U^{\prime \prime}(0)\right| / 2\right)^{1 / 2} x \sqrt{1-(1 / 3)\left|U^{\prime \prime}(0)\right|^{-1} U^{\prime \prime \prime}(0) x-\ldots} \\
& =\left(\left|U^{\prime \prime}(0)\right| / 2\right)^{1 / 2} x\left(1-(1 / 6)\left|U^{\prime \prime}(0)\right|^{-1} U^{\prime \prime \prime}(0) x-\ldots\right)
\end{aligned}
$$

and

$$
\begin{aligned}
g(y) & \left.\left.=\left|U^{\prime \prime}(0)\right| / 2\right)^{-1 / 2} y\left(1-(1 / 6)\left|U^{\prime \prime}(0)\right|^{-1} U^{\prime \prime \prime}(0)\left|U^{\prime \prime}(0)\right| / 2\right)^{-1 / 2} y-\ldots\right) \\
& \left.=\left|U^{\prime \prime}(0)\right| / 2\right)^{-1 / 2} y-(1 / 3)\left|U^{\prime \prime}(0)\right|^{-2} U^{\prime \prime \prime}(0) y^{2} \ldots,
\end{aligned}
$$

which is the expansion of $g(y)$ into the Taylor series at $y=0$. Using this expansion in (3.17) we obtain

$$
H(z ; b)-H(z ; 0)=b U^{\prime \prime \prime}(0) H_{1}(z)+b^{2} H_{2}(z ; b),
$$

where $H_{1}(z)$ and $H_{2}(z ; b)$ are $C^{\infty}$ smooth. From (3.19) we obtain now

$$
\zeta(y)=1+z+b U^{\prime \prime \prime}(0) h_{1}(z)+b^{2} h_{2}(z ; b),
$$

where $h_{1}(z)$ and $h_{2}(z ; b)$ are $C^{\infty}$ smooth. This obviously implies that $\forall A>1 \exists C=C(A)>0$ such that (3.12) holds when $0 \leqq y \leqq A$. Assume now that $y>A$ where $A>1$ is a large number.

By (3.9),

$$
\begin{aligned}
G(\zeta) & =\int_{1}^{\zeta}\left|\xi^{2}-1\right|^{1 / 2} d \xi=(1 / 2) \zeta\left(\zeta^{2}-1\right)^{1 / 2}-(1 / 2) \ln \left(\left(\zeta^{2}-1\right)^{1 / 2}+\zeta\right) \\
& =(1 / 2) \zeta^{2}-(1 / 2) \ln \zeta+(1 / 4)+\sum_{j=1}^{\infty} a_{j} \zeta^{-2 j}
\end{aligned}
$$

which implies that

$$
\begin{aligned}
H(\zeta) & \equiv(2 G(\zeta))^{1 / 2}=\zeta\left(1-\zeta^{-1} \ln 2 \zeta+(2 \zeta)^{-1}+\sum_{j=1}^{\infty} 2 a_{j} \zeta^{-2 j-1}\right)^{1 / 2} \\
& =\zeta+\sum_{j=2}^{\infty} \sum_{k=0}^{j} a_{j k} \zeta^{-j} \ln ^{k} \zeta
\end{aligned}
$$


where the series converges for large $\zeta$ and defines $H(\zeta)$ as an analytic function. Hence

$$
H^{-1}(y)=y+\sum_{j=2}^{\infty} \sum_{k=0}^{j} c_{j k} y^{-j} \ln ^{k} y .
$$

Now,

$$
g^{\prime}(b \xi)=g^{\prime}(0)+(b \xi) g^{\prime \prime}(0)+(b \xi)^{2} h(b \xi),
$$

where $h(t)$ is a smooth function, so from (3.14) we obtain that

$$
G(y ; b)=G(y)+b U^{\prime \prime \prime}(0) k_{0}(y)+b^{2} k(y ; b),
$$

where $k_{0}(y)$ and $k(y ; b)$ satisfy the estimates

$$
\left|\frac{d^{j} k_{0}(y)}{d y^{j}}\right| \leqq C_{j} y^{3-j}, \quad\left|\frac{d^{j} k(y ; b)}{d y^{j}}\right| \leqq C_{j} y^{4-j} .
$$

Assume now that $b y \leqq \delta$, where $\delta>0$ is a sufficiently small number so that

$$
\left(y^{2} \sim\right) G(y) \gg b k_{0}(y) \sim b y^{3}
$$

and

$$
G(y) \gg b^{2} k(y ; b) \sim b^{2} y^{4} .
$$

Then

$$
\begin{aligned}
H(y ; b) & \equiv(2 G(y ; b))^{1 / 2}=\left(2 G(y)+2 b U^{\prime \prime \prime}(0) k_{0}(y)+2 b^{2} k(y ; b)\right)^{1 / 2} \\
& =(2 G(y))^{1 / 2}+b U^{\prime \prime \prime}(0) K_{0}(y)+b^{2} K(y ; b) \\
& =H(y)+b U^{\prime \prime \prime}(0) K_{0}(y)+b^{2} K(y ; b),
\end{aligned}
$$

where $K_{0}(y)$ and $K(y ; b)$ satisfy the estimates

$$
\left|\frac{d^{j} K_{0}(y)}{d y^{j}}\right| \leqq C_{j} y^{2-j}, \quad\left|\frac{d^{j} K(y ; b)}{d y^{j}}\right| \leqq C_{j} y^{3-j} .
$$

Now,

$$
\begin{aligned}
\zeta(y ; b) & =H^{-1}(H(y ; b))=H^{-1}\left(H(y)+b U^{\prime \prime \prime}(0) K_{0}(y)+b^{2} K(y ; b)\right) \\
& =y+b U^{\prime \prime \prime}(0) L_{0}(y)+b^{2} L(y ; b)
\end{aligned}
$$

where

$$
\left|\frac{d^{j} L_{0}(y)}{d y^{j}}\right| \leqq C_{j} y^{2-j}, \quad\left|\frac{d^{j} L(y ; b)}{d y^{j}}\right| \leqq C_{j} y^{3-j} .
$$

This implies (3.12). So (3.12) is proved when $A<y$ and $b y \leqq \delta$. Similar estimates holds for $y<0$, so (3.12) is valid for all $y$ with $|b y| \leqq \delta$.

By (3.3)

$$
\varphi(x ; E)=2|a|^{1 / 2} \zeta\left(\left(U_{\max }-E\right)^{-1 / 2} f(x)\right),
$$

and (3.12) implies (2.14) and (2.15) for all $x$ with $|f(x)| \leqq \delta$. For $x$ with $|f(x)|>\delta$ (2.14) and (2.15) follow from (3.2) since then $E-U(x)>\delta_{0}$ and $a+\left(\varphi^{2} / 4\right)>\delta_{0}$ with some $\delta_{0}>0$. Thus in the case $E<U_{\max }$ Lemma 2.2 is proved. 
If $E=U_{\max }, a=0$ and (2.5) reduces to

$$
\left(2 \varphi^{\prime} \varphi\right)^{2}=f(x)^{2} \equiv 32 m\left(U_{\max }-U(x)\right),
$$

hence

$$
\varphi\left(x ; U_{\max }\right)=\left(\int_{0}^{x} f(\xi) d \xi\right)^{1 / 2}
$$

is a smooth solution of (2.5) and it satisfies (2.14) and (2.15).

If $E>U_{\max }$, the change of variables (3.3) reduces (3.2) to

$$
\zeta^{\prime}\left|\zeta^{2}+1\right|^{1 / 2}=h(y ; E)\left|y^{2}+1\right|^{1 / 2}
$$

and we define $\zeta$ as a solution of the equation

$$
G(\zeta)=G(y ; b)
$$

where $b=\left|E-U_{\max }\right|^{1 / 2}$ and

$$
G(y ; b)=\int_{0}^{y} h(\xi ; E)\left|\xi^{2}+1\right|^{1 / 2} d \xi .
$$

Since $G(y ; b)$ is a smooth function of $y$ with $G^{\prime}(y ; b)>0$, the solution $\zeta=\zeta(y ; b)$ is smooth in $y$ as well, and $\zeta^{\prime}(y ; b)>0$. The same arguments as we used in the case $a<0$ prove (2.14) and (2.15). Lemma 2.2 is proved.

Proof of Lemma 2.4. If we substitute $\psi_{+}$into (2.29) we obtain Eq. (2.3) with $\left.U+\left(\hbar^{2} / 2 m\right)\right) S\left(\varphi^{\prime}\right)$ instead of $U$. With this replacement (2.3) reduces to (2.5). Since by construction $\varphi$ is a solution of (2.5), $\psi_{+}$satisfies (2.29). Lemma 2.4 is proved.

Proof of Lemma 2.5. Assume (3/8) $L<x<(5 / 8) L$. As was mentioned $r$ comes from differentiation of $\chi$ in (2.28). We can differentiate $\chi$ either once or twice. If we differentiate $\chi$ twice we obtain the term

$$
\left.\left(\hbar^{2} / 2 m\right)\right) \chi^{\prime \prime}(x-(L / 2))\left[\psi_{+}(x ; E)-\psi_{+}(x-L ; E)\right] .
$$

Now, by (2.24),

$$
\begin{aligned}
\psi_{+}(x-L ; E) & =\frac{\hbar^{1 / 4}}{\sqrt{p(x-L ; E)+O\left(\hbar^{2}\right)}} \cos \left(\hbar^{-1} \int_{x_{-}}^{x-L} p(\xi ; E) d \xi+\phi_{+}(a)+O(\hbar)\right) \\
& =\frac{\hbar^{1 / 4}}{\sqrt{p(x ; E)+O\left(\hbar^{2}\right)}} \cos \left(\hbar^{-1} \int_{x_{+}}^{x} p(\xi ; E) d \xi-\phi_{+}(a)+O(\hbar)\right),
\end{aligned}
$$

hence comparing this with (2.23) we have

$$
\psi_{+}(x-L ; E)=\psi_{+}(x ; E)+O\left(\hbar^{1+(1 / 4)}\right),
$$

so that the term $(3.28)$ is $O\left(\hbar^{3+(1 / 4)}\right)$. In the same manner we estimate the term which appears when we differentiate $\chi$ once, as $O\left(\hbar^{3+(1 / 4)}\right)$. Since by $(2.23)$ $\|\psi\| \geqq C^{-1} \hbar^{1 / 4}$ (observe that the proof of (2.33) does not use Lemma 2.5) (2.31) follows. Lemma 2.5 is proved.

Proof of Lemma 2.6. Comparing (2.4) with the estimates (2.14) and (2.15) we obtain

$$
\left|S\left(\varphi^{\prime}\right)\right| \leqq C \tau \text {. }
$$


By (2.29) and (2.30)

$$
(H-E) \psi=-\hbar^{2} S\left(\varphi^{\prime}\right) \psi+r,
$$

hence (2.31) and (3.29) imply

$$
\|(H-E) \psi\| \leqq C\left(\tau \hbar^{2}+\hbar^{3}\right)\|\psi\|,
$$

so that (2.32) holds.

To prove $(2.33)$ observe that $\psi_{+}^{(\text {per })}(x ; E)$ with $\psi_{+}(x ; E)$ on the interval (1/ 8) $L<x<(3 / 8) L$, and $(2.23)$ gives

$$
\int_{(1 / 8) L}^{(3 / 8) L}\left|\psi_{+}(\xi ; E)\right|^{2} d \xi \geqq C^{-1} \hbar^{1 / 2},
$$

hence

$$
\left\|\psi_{+}^{(\text {per })}(x ; E)\right\|^{2} \geqq \int_{(1 / 8) L}^{(3 / 8) L}\left|\psi_{+}(\xi ; E)\right|^{2} d \xi \geqq C^{-1} \hbar^{1 / 2},
$$

and thus (2.33) is proved.

Proof of Lemma 2.7. Let

$$
\psi=\sum_{k=1}^{\infty} a_{k} \psi_{k}
$$

be an expansion of $\psi=\psi_{+}^{(\text {per })}(x ; E)$ into series in eigenfunctions of the operator $H$. Let

$$
\Delta E=\min _{k}\left|E-E_{k}\right|
$$

Then

$$
\|(H-E) \psi\|=\left\|\sum_{k}\left(E_{k}-E\right) a_{k} \psi_{k}\right\| \geqq \Delta E\|\psi\|,
$$

hence (2.32) implies

$$
\Delta E \leqq C \tau \hbar^{2}
$$

If $\left|E-U_{\max }\right| \leqq \hbar^{4 / 3}$ and $\psi=\psi_{+}^{(\text {per })}\left(x ; U_{\max }\right)$, then

$$
\|(H-E) \psi\| \leqq\left\|\left(H-U_{\max }\right) \psi\right\|+\hbar^{4 / 3}\|\psi\| \leqq\left(C \hbar^{2}+\hbar^{4 / 3}\right)\|\psi\|,
$$

and we obtain

$$
\Delta E \leqq C \hbar^{4 / 3}
$$

hence (2.34) follows.

Let us prove that $\psi_{m,+}$ has exactly $2 k$ zeroes. Consider some point $x_{0}$ near $L / 2$ where

$$
\omega \equiv(2 \pi)^{-1}\left(\hbar^{-1} \int_{x_{+}}^{x} p(\xi ; E) d \xi-\phi_{+}(a)\right)
$$

is integer, $\omega=l$. Then (2.33)-(2.25) together with Lemma 2.3 imply that $\psi_{+}(x ; E)$ has exactly $2 k$ zeroes on the interval $\left[x_{0}-L, x_{0}\right]$. Moreover, the same is true for 
$\psi_{+}(x ; E+\Delta E)$ if $0 \leqq \Delta E \leqq \hbar^{5 / 4}$, because then

$$
0<\hbar^{-1} \int_{\left.x_{+}(E+\Delta E)\right)}^{x} p(\xi ; E+\Delta E) d \xi-\hbar^{-1} \int_{x_{+}(E)}^{x} p(\xi ; E) d \xi \leqq C \hbar^{1 / 12}
$$

and the argument in cosine in (2.23) changes too little when we pass from $E$ to $E+\Delta E$ to gain more zeroes in $\psi_{+}(x ; E+\Delta E)$ than we have in $\psi_{+}(x ; E)$.

Observe now that by Lemma $2.4, \psi_{+}(x ; E+\Delta E)$ satisfies the equation

$$
\psi^{\prime \prime}+q_{0} \psi=0
$$

with

$$
q_{0}=\left(2 m / \hbar^{2}\right)\left[E+\Delta-U-\left(\hbar^{2} /(2 m)\right) S\left(\varphi^{\prime}\right)\right],
$$

and $\psi=\psi_{m,+}(x)$ satisfies the equation

$$
\psi^{\prime \prime}+q \psi=0
$$

with

$$
q=\left(2 m / \hbar^{2}\right)(E-U) .
$$

Assume $\left|E-U_{\max }\right|>\hbar^{4 / 3}$. Then by Lemma $2.2 S\left(\varphi^{\prime}\right) \leqq C \hbar^{-2 / 3}$ and if we take $\Delta E=\hbar^{5 / 4}$, then we get $q_{0}>q$. In this case by the "argument principle" any solution of (3.31) cannot have more zeroes than the number of zeroes of any solution of (3.30) plus 1 (see, e.g., [Ble]). Hence $\psi_{m,+}(x)$ has at most $2 k+1$ zeroes on $\left[x_{0}-L, x_{0}\right]$. All these zeroes are simple since $\psi_{m,+}$ satisfies $(2.36)$ which is a linear equation of the second order. Since $\psi_{m,+}$ is periodic and all its zeroes are simple, $\psi_{m,+}$ has an even number of zeroes so this number does not exceed $2 k$. A similar reasoning in which we use $\psi_{+}(x ; E-\Delta E)$ instead of $\psi_{+}(x ; E+\Delta E)$, shows that the number of zeroes of $\psi_{m,+}$ is not smaller than $2 k$, hence this number is equal to $2 k$.

If $\left|E-U_{\max }\right| \leqq \hbar^{4 / 3}$ we use the same considerations with $\psi=\psi_{+}^{(\text {per })}\left(x ; U_{\max }\right)$ instead of $\psi_{+}^{\text {(per) }}(x ; E)$, and we again obtain that the number of zeroes of $\psi_{m,+}$ is $2 k$. Lemma 2.7 is proved.

Proof of Lemma 2.8. Assume $\psi_{m,+}$ and $\psi_{m,-}$ are collinear. Then $E_{m,+}=E_{m,-} \equiv E$. Let $E_{ \pm}$be solutions of (1.17). Then by (2.34)

$$
\left|E-E_{ \pm}\right| \leqq C \tau \hbar^{2}
$$

(we assume for a moment that $\left|E-U_{\max }\right|>\hbar^{4 / 3}$ ), hence

$$
\left|E_{+}-E_{-}\right| \leqq C \tau \hbar^{2} \text {. }
$$

Hence Lemma 2.9 and (1.7) imply

$$
\left|\gamma_{+}-\gamma_{-}\right| \leqq C \tau \hbar^{3 / 4}, \quad \gamma_{ \pm}=\gamma_{ \pm}\left(E_{ \pm}\right)
$$

By (2.33)

$$
\psi_{+}\left(x ; E_{+}\right)=\frac{\hbar^{1 / 4}}{\sqrt{p\left(x ; E_{+}\right)+O\left(\hbar^{2}\right)}} \cos \left(\hbar^{-1} \int_{x_{+}}^{x} p\left(\xi ; E_{+}\right) d \xi-(\pi / 2) \gamma_{+}+O(\hbar)\right) \text {. }
$$


For $\psi_{-}\left(x ; E_{-}\right)$we have a similar formula:

$$
\psi_{-}\left(x ; E_{+}\right)=\frac{\hbar^{1 / 4}}{\sqrt{p\left(x ; E_{-}\right)+O\left(\hbar^{2}\right)}} \sin \left(\hbar^{-1} \int_{x_{+}}^{x} p\left(\xi ; E_{-}\right) d \xi-(\pi / 2) \gamma_{-}+O(\hbar)\right)
$$

This enables us to estimate the scalar product of $\psi_{+}\left(x ; E_{+}\right)$and $\psi_{-}\left(x ; E_{-}\right)$as

$$
\left|\int_{-L / 2}^{L / 2} \psi_{+}\left(x ; E_{+}\right) \psi_{-}\left(x ; E_{-}\right) d x\right| \leqq C \tau \hbar^{5 / 4},
$$

which implies that $\psi_{+}\left(x ; E_{+}\right)$and $\psi_{-}\left(x ; E_{-}\right)$are almost perpendicular (the angle in $L^{2}$ between $\psi_{+}\left(x ; E_{+}\right)$and $\psi_{-}\left(x ; E_{-}\right)$is $\left.O\left(\tau \hbar^{1 / 4}\right)\right)$. Hence $\psi_{ \pm} \equiv \psi_{+}^{(\text {per })}\left(x ; E_{ \pm}\right)$are almost perpendicular as well.

On the other hand $\| H-E) \psi_{ \pm}\left\|\leqq C \hbar^{4 / 3}\right\| \psi_{ \pm} \|$. Since $\psi_{ \pm}$are almost perpendicular, at least one of them has a "big" projection $\psi$ on the orthogonal complement to the eigenfunction $\psi_{m,+}$. Then $\|(H-E) \psi\|<C \hbar^{4 / 3}\|\psi\|$, hence there is an eigenfunction $\psi^{\prime}$ in this orthogonal complement with an eigenvalue $E^{\prime}$ which differs from $E$ in $O\left(\hbar^{4 / 3}\right)$. Now we can take $\psi_{m,-}=\psi^{\prime}$ and $E \psi_{m,-}=E^{\prime}$.

Lemma 2.8 is proved.

Acknowledgements. The author thanks Michael Berry, Freeman Dyson, Charles Fefferman, Evans Harrell, Tom Spencer and Alejandro Uribe for useful discussions of the article. The author is grateful to the Institute for Advanced Study, Princeton, where this work was done, for financial support. The work is supported by a grant from the Ambrose Monell Foundation and by a grant in aid \# DE-FG02-90ER40542 from the US Department of Energy.

\section{References}

[BE] Bateman, H., Erdelyi, A.: Higher transcendental functions. New York: McGraw-Hill 1953

[BM] Berry, M.V., Mount, K.E.: Semiclassical approximation in wave mechanics. Rep. Progr. Phys. 35, 315-397 (1972)

[BT] Berry, M.V., Tabor, M.: Closed orbits and the regular bound spectrum. Proc. R. Soc. Lond. A349, 101-123 (1976)

[Ble] Bleher, P.M.: Quasi-classical expansion and the problem of quantum chaos, Lect. Notes Math. 1469, 60-89 (1991)

[Din] Dingle, R.B.: Appl. Sci. Res. B5, 345-367 (1956)

[FHWW] Ford, K.W., Hill, D.L., Wakano, M., Wheeler, J.A.: Quantum effects near a barrier maximum. Ann. Phys. N.Y., 7, 239 (1959)

[FF] Fröman, N., Fröman, P.O.: JWKB approximation. Amsterdam: North-Holland, 1965

[FFMP] Fröman, N., Fröman, P.O., Muhrman, U., Paulsson, R.: On the quantal treatment of the double-well potential problem by means of certain phase-integral approximation. Ann. Phys. N.Y. 74, 314-323 (1972)

[HM] Hecht, C.E., Mayer, J.E.: Extension of the WKB equation. Phys. Rev. 106, 1156-1160 (1957)

[KMS] Kosygin, D.V., Minasov, A.A., Sinai, Y.G.: Statistical properties of the LaplaceBeltrami operators on the Liouville surfaces. Uspechi Matem. Nauk 48, 3-130 (1993)

[Lan] Langer, R.: On the asymptotic solutions of ordinary differential equations, with an application to the Bessel functions of large order. Trans. Am. Math. Soc. 33, 23-64 (1931

[Laz] Lazutkin, V.F.: Quasiclassical asymptotics of eigenfunctions. In: Modern problems of mathematics. Fundamental trends. (Itogi nauki i techniki). 34, VINITI of the USSR Acad. of Sci., 1989 
[MG] Miller, S.C., Good, R.H., Jr.: A WKB approximation to the Schrödinger equation. Phys. Rev. 91, 174-179 (1953)

[Mil] Miller, W.H.: Semiclassical treatment of multiple turning-point problem - phase shifts and eigenvalues. J. Chem. Phys. 48, 1651-1668 (1968)

[Mor] Moriguchi. H.: An improvement of the WKB method in the presence of turning points and the asymptotic solutions of a class of Hill equations. J. Phys. Soc. Japan 14, 1771-1796 (1959)

[Olv1] Olver, F.W.J.: Uniform asymptotic expansions for Weber parabolic cylinder functions of larger orders. J. Res. Nat. Bur. Standards Sect. 63, 131-169 (1959)

[Olv2] Olver, F.W.J.: Error bounds for asymptotic expansions, with application to cylinder functions of large argument. In: Asymptotic solutions of differential equations and their applications. Ed. C.H. Wilcox, pp. 163-183, New York: Wiley, 1964

[Olv3] Olver, F.W.J.: Asymptotics and special functions. New York e.a.: Academic Press 1974

[Sch] Schwid, N.: The asymptotic forms of the Hermite and Weber functions. Trans. Am. Math. Soc. 37, 339-362 (1935)

[WW] Whittaker, E.T., Watson, G.N.: A course of modern analysis. London: Cambridge Univ. Press, 1946

Communicated by B. Simon 